\title{
Controlled-intensity detection peaks in a binary joint transform correlator
}

\author{
Arturo Carnicer, Santiago Vallmitjana, Ignacio Juvells, and J. R. de F. Moneo
}

\begin{abstract}
In multiobject pattern recognition the height of the correlation peaks should be controlled when the power spectrum of a joint transform correlator is binarized. In this paper a method to predetermine the value of detection peaks is demonstrated. The technique is based on a frequency-variant threshold in order to remove the intraclass terms and on a suitable factor to normalize the binary joint power spectrum. Digital simulations and experimental hybrid implementation of this method were carried out.

Key words: Optical correlation, real-time pattern recognition, nonlinear effects.
\end{abstract}

\section{Introduction}

Since the inception of the joint transform correlator, ${ }^{1}$ hybrid architectures have been proposed for real-time image recognition. ${ }^{2}$ For an increase in the recognition capability the binarization of the joint power spectrum (JPS) has been suggested. ${ }^{3}$ This procedure permits us to obtain higher correlation-peak intensity and small sidelobes.

The suitable choice of a binarization threshold is a problem that has been widely investigated. ${ }^{4-7}$ Also, one of the problems analyzed on multiobject binary joint transform correlators is the presence of false peaks created by the harmonics of low-frequency contents. ${ }^{8,9}$ One possible way of alleviating this difficulty is by the use of a suitable binarization threshold value. ${ }^{10}$

Another problem involved in detection by means of a binary joint transform correlator is the lack of control over the value of the recognition peaks. In this paper we analyze by digital simulation a method that predetermines the value of single detection peaks in scenes containing several different objects using a binary joint transform correlator. To guarantee the success of the method, one should use the frequency-variant threshold in order to remove the intraclass terms. ${ }^{6,11} \mathrm{~A}$ hybrid implementation of this method was carried out to corroborate the results obtained in the simulation.

The authors are with the Laboratori d'Optica, Universitat de Barcelona, Department de Física Aplicada i Electrònica, Diagonal 647, Barcelona E-08028, Spain.

Received 6 July 1993; revised manuscript received 27 September 1993.

0003-6935/94/143070-06\$06.00/0.

(1) 1994 Optical Society of America.

\section{Background}

The JPS of a joint transform correlator is described by the following equation:

$$
\begin{aligned}
I(u, v)= & \left|H_{R}(u, v)\right|^{2}+|H(u, v)|^{2} \\
& +2\left|H_{R}(u, v) \| H(u, v)\right| \\
& \times \cos \left[x_{0} u+y_{0} v+\phi_{s}(u, v)-\phi_{R}(u, v)\right],
\end{aligned}
$$

where $\left|H_{R}(u, v)\right| \exp \left[i \phi_{R}(u, v)\right]$ and $|H(u, v)| \exp \left[i \phi_{s}\right.$ $(u, v)]$ are the Fourier transforms of the reference, $h_{R}(x, y)$, and the scene, $h(x, y)$, respectively. The scene is located at $\left(x_{0}, y_{0}\right)$.

The binary joint power spectrum (BJPS), $I_{b}(u, v)$ is obtained by assignment of the values +1 or -1 to $I(u, v)$ following Eq. (2):

$$
\begin{array}{ll}
I_{b}(u, v)=1, & \text { for } I(u, v)>I_{T}, \\
I_{b}(u, v)=-1, & \text { for } I(u, v)<I_{T} .
\end{array}
$$

$I_{T}$ is a predetermined threshold value. This bipolar function can be expressed as a Fourier expansion ${ }^{4}$ :

$$
\begin{aligned}
I_{b}(u, v)= & \sum_{\nu} A_{v}(u, v) \\
& \times \cos \left\{v\left[x_{0} u+y_{0} v+\phi_{s}(u, v)-\phi_{R}(u, v)\right]\right\},
\end{aligned}
$$

where coefficients $A_{\nu}(u, v)$ are described by

$$
\begin{aligned}
A_{v}(u, v)= & (2 / \pi v) \sin \left(v \operatorname { a r c c o s } \left\{\left[I_{T}-\left|H_{R}(u, v)\right|^{2}\right.\right.\right. \\
& \left.\left.\left.-|H(u, v)|^{2}\right] / 2\left|H_{R}(u, v) \| H(u, v)\right|\right\}\right) .
\end{aligned}
$$

By using variable threshold ${ }^{6,11} I_{T}(u, v)=\left|H_{R}(u, v)\right|^{2}+$ 


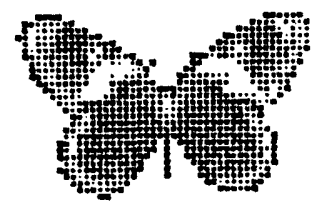

(a)
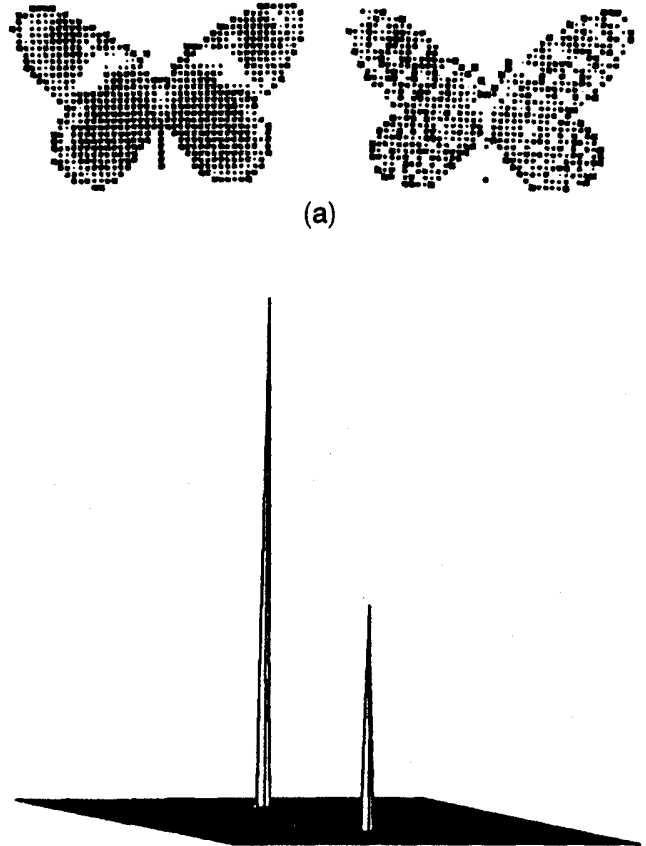

(b)

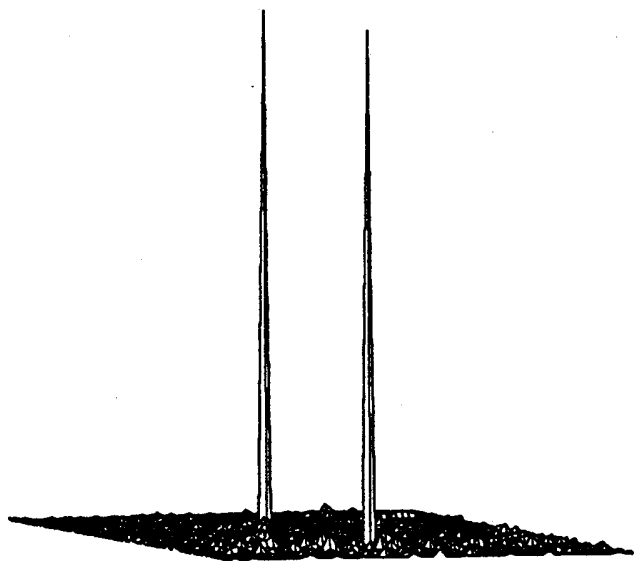

(c)

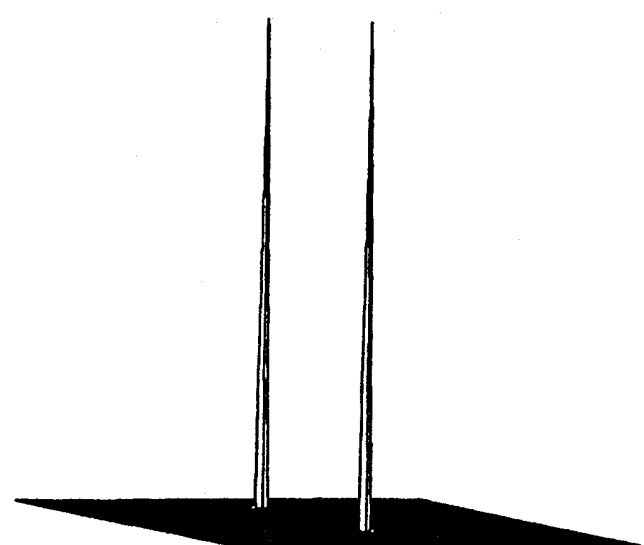

(d)

Fig. 1. (a) Butterflies (scene 1), (b) peaks before normalization, (c) normalized peaks with $W_{R}(u, v)=\left|H_{R}(u, v)\right|^{-1}$, (d) normalized peaks with $W_{R}=\operatorname{med}\left[\left|H_{R}(u, v)\right|^{2}\right]^{-1 / 2}$.

\begin{tabular}{lcc}
\hline & \multicolumn{2}{c}{ Value (\%) } \\
\cline { 2 - 3 } \multicolumn{1}{c}{ Normalizing Function } & Butterfly 1 & Butterfly 2 \\
\hline Without normalization & 100 & 45 \\
$W_{R}(u, v)=\left|H_{R}(u, v)\right|^{-1}$ & 100 & 100 \\
$W_{R}=\operatorname{med}\left[\left|H_{R}(u, v)\right|^{2}\right]^{-1 / 2}$ & 99 & 100 \\
\hline
\end{tabular}

$|H(u, v)|^{2}$, we can write the BJPS in Eq. (3) as

$$
\begin{aligned}
I_{b} & (u, v) \\
= & \sum_{\nu \text { odd }}(2 / \pi v)(-1)^{(\nu-1) / 2} \\
& \quad \times \cos \left\{\nu\left[x_{0} u+y_{0} v+\phi_{S}(u, v)-\phi_{R}(u, v)\right]\right\} \\
= & (2 / \pi) \cos \left[x_{0} u+y_{0} v+\phi_{S}(u, v)-\phi_{R}(u, v)\right]+\cdots .
\end{aligned}
$$

Higher terms $(v>1)$ can be removed by spatial filtering, and only the first, $\left[I_{b}^{(1)}(u, v)\right]$, is taken into account. If the reference is equal to the scene, then $\phi_{S}(u, v)=\phi_{R}(u, v)$, and consequently the correlation plane can be described by

$$
\mathrm{FT}\left[I_{b}^{(1)}(u, v)\right]=\delta\left(x+x_{0}, y+y_{0}\right)+\delta\left(x-x_{0}, y-y_{0}\right)
$$

where FT is the Fourier-transform operator.

If the scene is composed of several objects, $h(x, y)=$ $\Sigma_{j} h_{j}\left(x-x_{j}, y-y_{j}\right)$, and if the variable threshold is considered, the first term of the BJPS can be written as ${ }^{6}$ :

$$
\begin{aligned}
I_{b}^{(1)}(u, v)= & \sum_{j} I_{b j}^{(1)}(u, v), \\
I_{b, j}^{(1)}(u, v)= & (2 / \pi)\left[\left|H_{j}(u, v)\right| /|H(u, v)|\right] \\
& \times \cos \left[x_{j} u+y_{j} v+\phi_{j}(u, v)-\phi_{R}(u, v)\right],
\end{aligned}
$$

where $\left|H_{j}(u, v)\right| \exp \left[i \phi_{j}(u, v)\right]$ is the Fourier transform of the $j$ th object and $I_{b, j}^{(1)}(u, v)$ is the contribution of this to $I_{b}^{(1)}$. For $j=R, \phi_{j}(u, v)=\phi_{R}(u, v)$, and in this case.

$$
I_{b, R}^{(1)}(u, v)=(2 / \pi)\left[\left|H_{R}(u, v)\right| /|H(u, v)|\right] \cos \left(x_{j} u+y_{j} v\right) .
$$

By analyzing Eq. (7), we can conclude that the height of the correlation peak is $\mathrm{FT}\left[\left|H_{R}(u, v)\right| /|H(u, v)|\right]$, and consequently the value of the detection depends on the ratio of Fourier-transform amplitudes of the scene and the reference.

\section{Peak Normalization}

To avoid false detection or missing peaks, one should keep the height of recognition maxima the same. However, as shown in Section 2 and Ref. 6, peak value is reference and scene dependent. The aim of this paper is to demonstrate that well-controlled peak 
values may be obtained by use of a suitable weight function. As a consequence, advice that the detection threshold of a control that registers correlation can be programmed. Let $W_{R}(u, v)$ be the weight
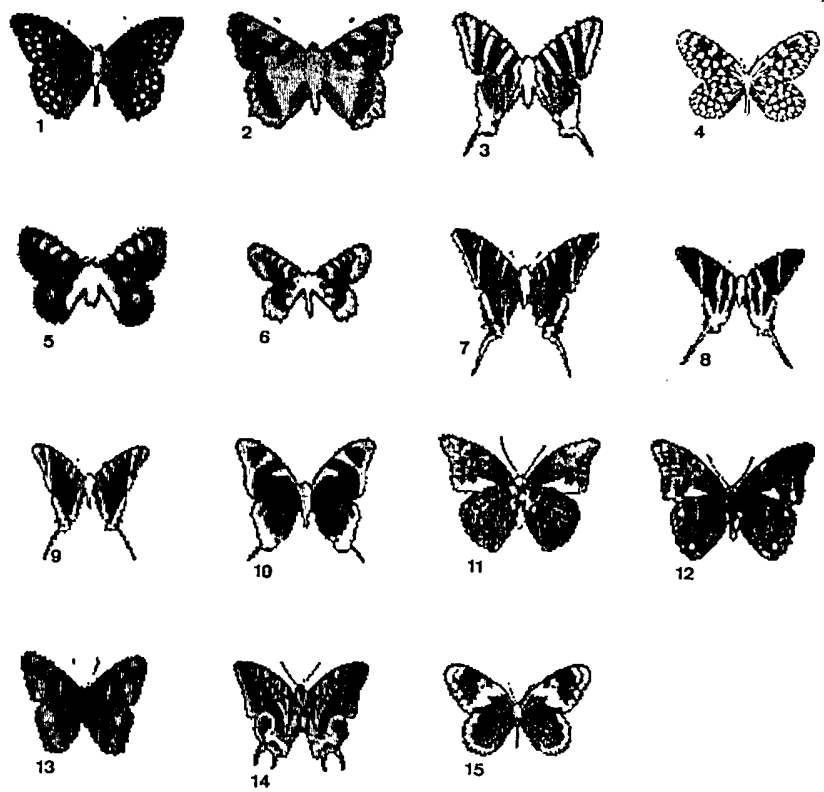

(a)

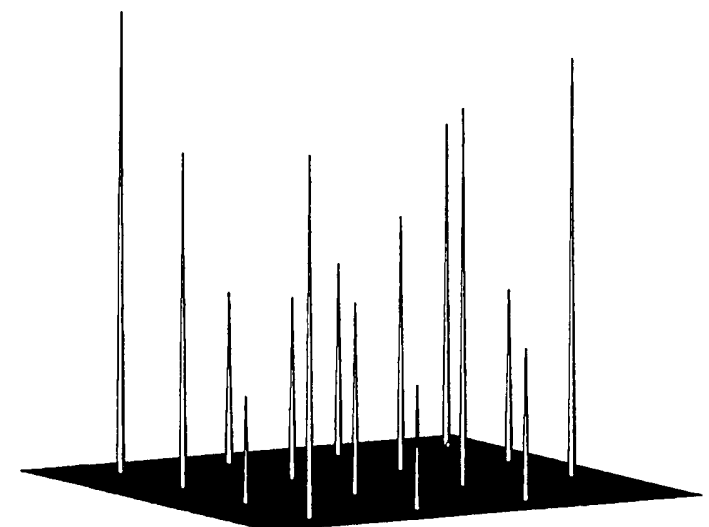

(b)

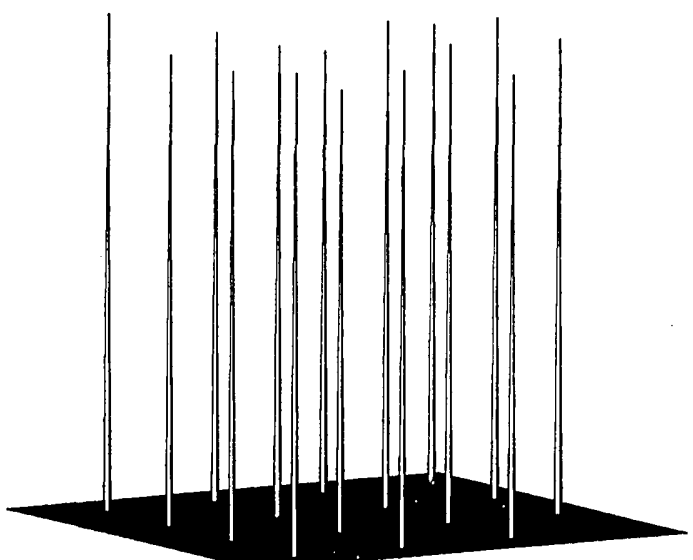

(c)

Fig. 2. (a) Butterflies (scene 2), (b) peaks before normalization, (c) normalized peaks. function of a BJPS; i.e.,

$$
\begin{aligned}
\hat{I}_{b}^{(1)}(u, v) & =W_{R}(u, v) I_{b}^{(1)}(u, v) \\
& =\sum_{j} W_{R}(u, v) I_{b, j}^{(1)}(u, v),
\end{aligned}
$$

where $\hat{I}$ is the $W_{R}$-normalized JPS.

A simple way to solve this problem is to take the weight function as $W_{R}(u, v)=\left|H_{R}(u, v)\right|^{-1}$. This can be obtained optically by registration of $\left|H_{R}(u, v)\right|^{2}$ and then by computation of $\left[\left|H_{R}(u, v)\right|^{2}\right]^{-1 / 2}$. Nevertheless, it is not realistic to try to obtain $W_{R}(u, v)$ in this way because some floating-point processes are involved. Another related problem is the possible zero values of $\left|H_{R}(u, v)\right|$ : at these spatial frequencies the modified JPS cannot be defined. Last, notice in this case that $\hat{I}_{b}^{(1)}$ is a nonbinary function, and thus a gray-level modulator should be used.

As an alternative that permits a direct hybrid implementation, we propose the use of weight as $W_{R}(u, v)=W_{R}=\operatorname{med}\left[\left|H_{R}(u, v)\right|\right]^{-1}$, where med is the median value of the Fourier-transform amplitude of $h_{R}(x, y)$. As is well known, the median value is one of the simplest statistical parameters for describing a value distribution. In the hypothesis that functions $\left|H_{j}(u, v)\right|$ are slowly variant, taking this weight is a good approximation that leads to well-normalized correlation peaks, as described in Sections 3 and 4.

The median of $\left|H_{R}(u, v)\right|$ can be directly obtained in a hybrid setup by registration of the intensity $\left|H_{R}(u, v)\right|^{2}$. Then it is easy to prove that

$$
\left\{\operatorname{med}\left[\left|H_{R}(u, v)\right|^{2}\right]\right\}^{1 / 2}=\operatorname{med}\left[\left|H_{R}(u, v)\right|\right] .
$$

Finally, the modified BJPS $\hat{I}_{b}^{(1)}(u, v)$ can be written as

$$
\begin{aligned}
\hat{I}_{b}^{(1)}(u, v) & =\sum_{j} W_{R} I_{b, j}^{(1)}(u, v) \\
& =\sum_{j}\left\{\operatorname{med}\left[\left|H_{R}(u, v)\right|^{2}\right]\right\}^{1 / 2} I_{b, j}^{(1)}(u, v) .
\end{aligned}
$$

\begin{tabular}{|c|c|c|c|c|c|}
\hline Butterfly & $\begin{array}{c}\text { Autocor- } \\
\text { relation } \\
\text { Value } \\
\left(C_{d}\right)\end{array}$ & $\begin{array}{c}\text { Value } \\
(\%)\end{array}$ & $\begin{array}{c}\text { Reference } \\
\text { Median } \\
\text { (med) }\end{array}$ & $C_{d} / \mathrm{med}$ & $\begin{array}{c}\text { Value } \\
(\%)\end{array}$ \\
\hline 1 & 8529 & 100.0 & 4.42 & 1931 & 100.0 \\
\hline 2 & 2874 & 33.7 & 1.58 & 1822 & 94.3 \\
\hline 3 & 3260 & 38.2 & 1.89 & 1720 & 89.1 \\
\hline 4 & 5616 & 65.8 & 3.14 & 1791 & 92.7 \\
\hline 5 & 5676 & 66.5 & 3.09 & 1836 & 95.1 \\
\hline 6 & 3037 & 35.6 & 1.65 & 1835 & 95.0 \\
\hline 7 & 4384 & 51.4 & 2.32 & 1891 & 97.9 \\
\hline 8 & 2872 & 33.7 & 1.54 & 1856 & 96.1 \\
\hline 9 & 1763 & 20.7 & 0.97 & 1821 & 94.3 \\
\hline 10 & 3196 & 37.5 & 1.86 & 1714 & 88.8 \\
\hline 11 & 6428 & 75.4 & 3.46 & 1857 & 96.2 \\
\hline 12 & 7060 & 82.8 & 3.82 & 1850 & 95.8 \\
\hline 13 & 6088 & 71.4 & 3.26 & 1867 & 96.7 \\
\hline 14 & 2106 & 24.7 & 1.14 & 1851 & 95.9 \\
\hline 15 & 2753 & 32.2 & 1.54 & 1792 & 92.8 \\
\hline
\end{tabular}

Table 2. Butterflies (Scene 2) 


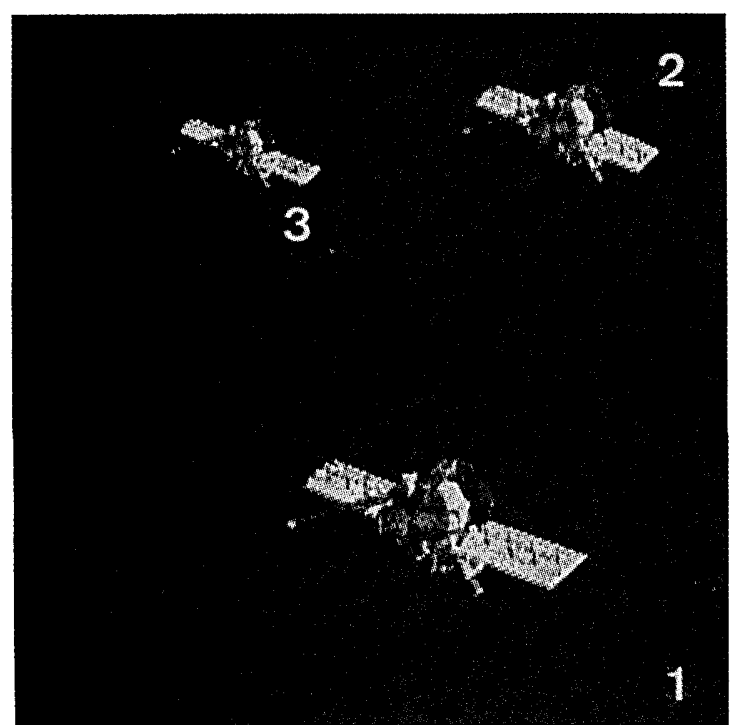

(a)

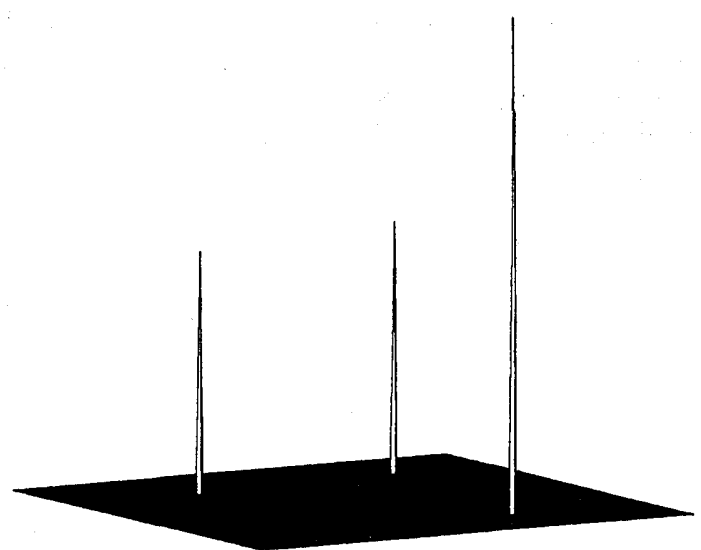

(b)

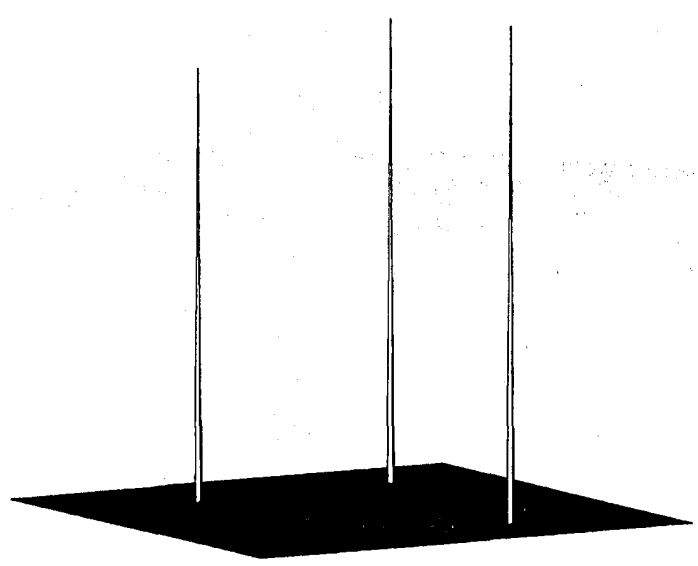

(c)

Fig. 3. (a) Satellite scene, (b) peaks before normalization, (c) normalized peaks.

Notice that factor $W_{R}$ is dependent only on the reference, and consequently it can be computed independently of the scene. For a set of references the associated $W_{R}$ value can be calculated and then used in any situation.

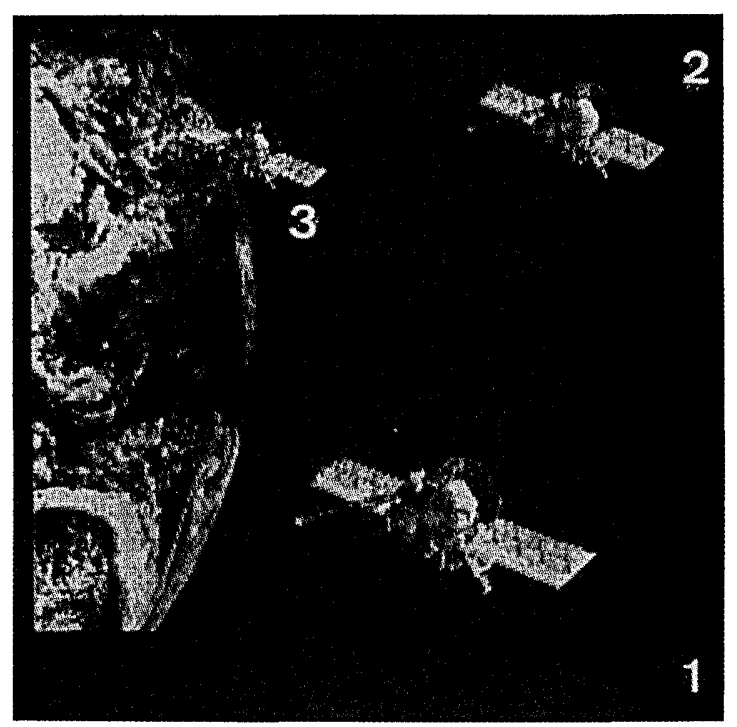

(a)

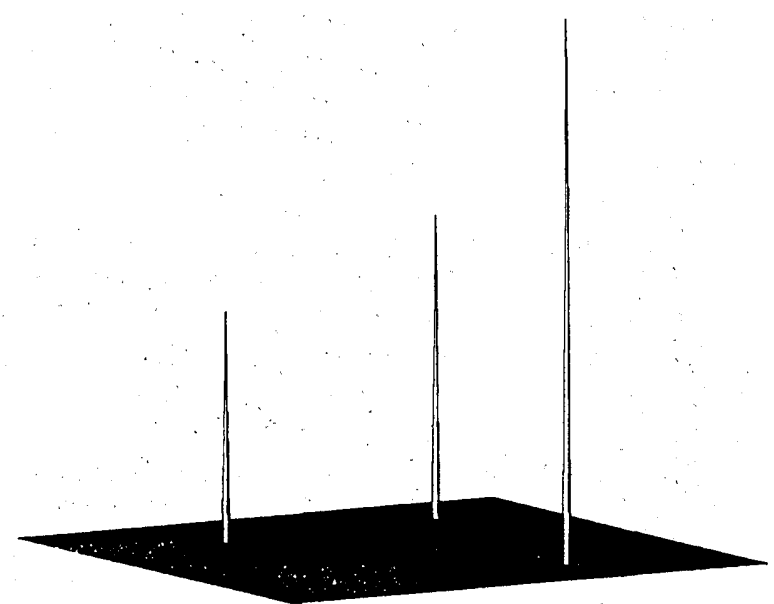

(b)

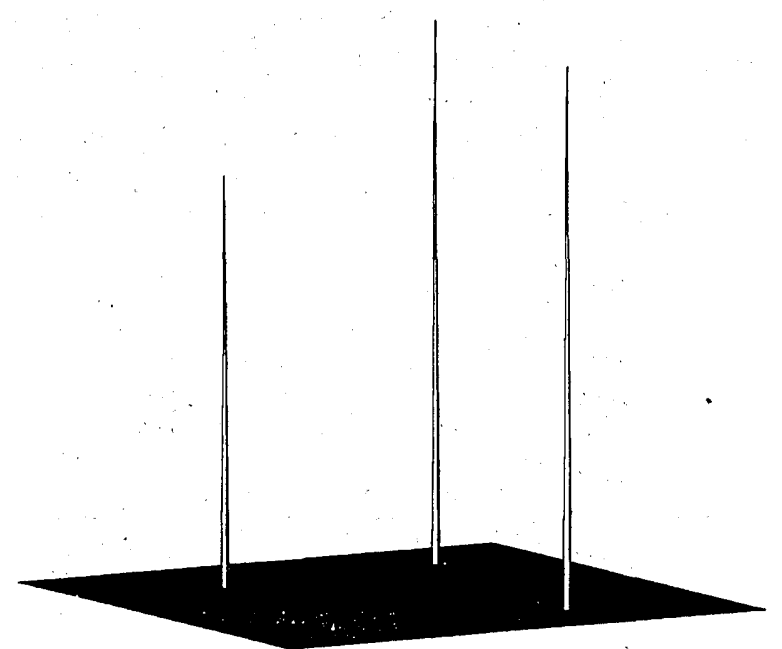

(c)

Fig. 4. (a) Satellite and Earth scene, (b) peaks before normalization, (c) normalized peaks. 
Table 3. Satellite Scene

\begin{tabular}{cccccc}
\hline & $\begin{array}{c}\text { Autocor- } \\
\text { relation } \\
\text { Value } \\
\text { Satellite }\end{array}$ & $\begin{array}{c}\text { Value } \\
\left(C_{d}\right)\end{array}$ & $\begin{array}{c}\text { Reference } \\
\text { Median } \\
(\%)\end{array}$ & $\begin{array}{c}\text { (med) } \\
C_{d} / \text { med }\end{array}$ & $\begin{array}{c}\text { Value } \\
(\%)\end{array}$ \\
\hline 1 & 36423 & 100.0 & 2.84 & 12831 & 100.0 \\
2 & 18393 & 50.5 & 1.55 & 11866 & 92.5 \\
3 & 17909 & 49.2 & 1.60 & 11213 & 87.4 \\
\hline
\end{tabular}

\section{Digital Simulation}

In order to show the suitability of the hypothesis introduced above, a series of digital simulations were carried out with different scenes. The first experiment consists of a demonstration that the approximation of height function $W_{R}(u, v)=\left|H_{R}(u, v)\right|^{-1}$ by normalizing constant $W_{R}=\operatorname{med}\left[\mid H_{R}(u, v)\right]^{-1}$ is appropriate in all the situations analyzed. Figure 1(a) shows a simple image composed by two gray-level butterflies, and correlation results appear in Fig. 1(b). Each peak is obtained in separate computations, but for conciseness these correlations are jointly displayed. As we can see in Fig. 1(b) and in Table 1, the height of the maxima are very dissimilar. Figure 1(c) displays the correlations obtained by application of the frequency-variant weight function, $W_{R}(u, v)$ : although the correlation plane is noisy, the values of the peaks are equal. Last, Fig. 1(d) shows the results obtained when the BJPS is renormalized with constant weight $W_{R}$. As can be seen, the correlation peaks are practically identical, showing good performance of the approximation.

In order to show that the use of $W_{R}$ to normalize the BJPS is satisfactory for a wide range of scenes, we present some results. Figure 2(a) is composed of 15 gray-level butterflies. Figures 2(b) and 2(c) show the superpositions of autocorrelations computed individually, as in Figs. 1(b)-1(d), before and after the normalization of their respective BJPS's. Table 2 presents the detailed values of the maxima (columns 2 and 3 ) give nonnormalized values; columns 5 and 6 give normalized values) and the height of $W_{R}$ (column 4). As can be seen, the most unfavorable case corresponds to butterfly 10 , the height of which is $88.8 \%$ of the highest one (butterfly 1). This result corroborates the hypothesis that $W_{R}$ is a good approximation to $W_{R}(u, v)$.

Figures 3(a)-3(c) and 4(a)-4(c) correspond to the reproduction of the former operations with different scenes in order to show the general use of the method. Figure 3(a) is a scene composed of three satellites, and

Table 4. Satellite and Earth Scene

\begin{tabular}{|c|c|c|c|c|c|}
\hline Satellite & $\begin{array}{c}\text { Autocor- } \\
\text { relation } \\
\text { Value } \\
\left(C_{d}\right) \\
\end{array}$ & $\begin{array}{c}\text { Value } \\
(\%)\end{array}$ & $\begin{array}{c}\text { Reference } \\
\text { Median } \\
\text { (med) }\end{array}$ & $C_{d} / \mathrm{med}$ & $\begin{array}{c}\text { Value } \\
(\%)\end{array}$ \\
\hline 1 & 11283 & 100.0 & 2.84 & 3975 & 98.8 \\
\hline 2 & 6230 & 55.2 & 1.55 & 4020 & 100.0 \\
\hline 3 & 4850 & 43.0 & 1.60 & 3037 & 75.5 \\
\hline
\end{tabular}

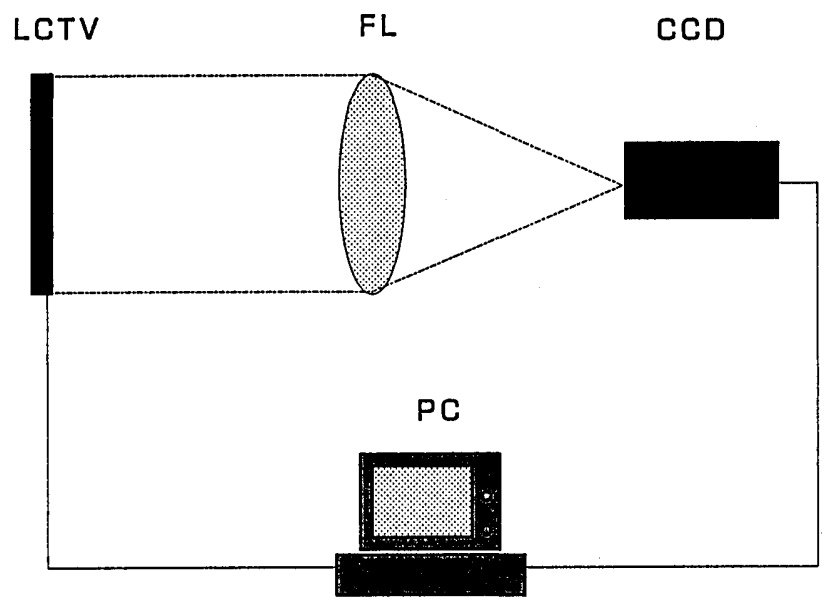

Fig. 5. Schematic diagram of the optical setup: FL, Fouriertransform lens; PC, personal computer.

Fig. 4(a) shows the same objects, one of which is affected by real background. Tables 3 and 4 detail the results obtained in these situations. As we can see, the heights of autocorrelations in both cases are satisfactory. Nevertheless, the presence of back-

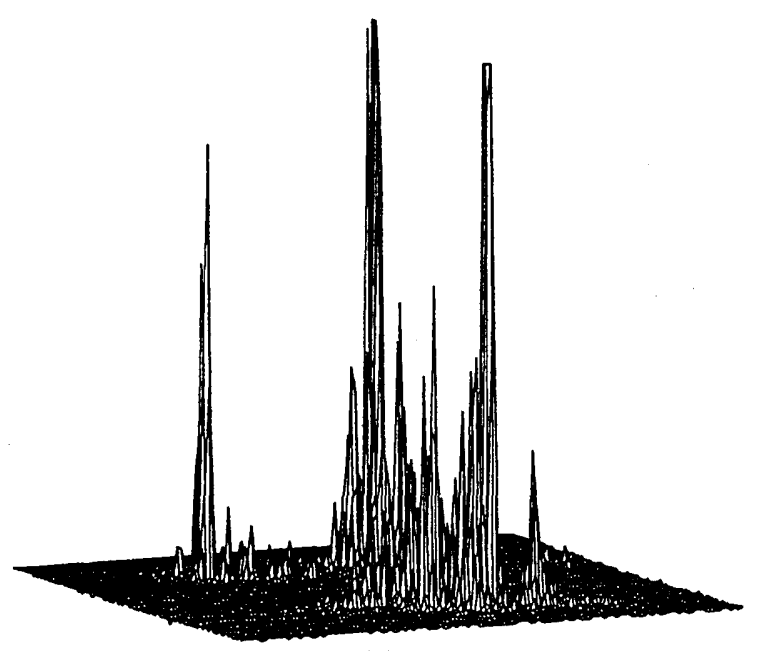

(a)

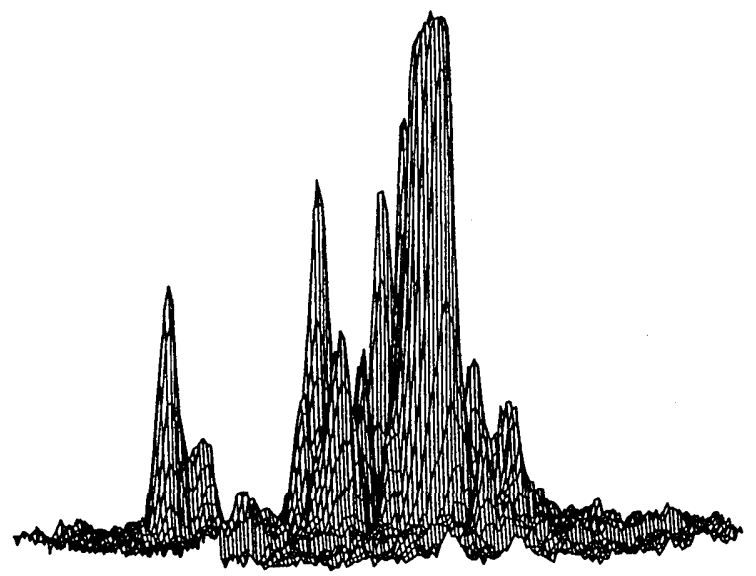

(b)

Fig. 6. Three-dimensional representation of optical correlation: (a) $\hat{I}_{\text {sum }}$ photographically recorded, (b) $\hat{I}_{\text {sum }}$ displayed on a LCTV. 
ground in the scene reduces the value of the peak corresponding to satellite $3(75 \%$ of peak 1$)$. However, this is not an obstacle to the detection of this object.

\section{Experiment}

In order to show the difficulties presented in the implementation of the method in a hybrid real-time setup, ${ }^{12}$ we carried out two preliminary cases of detection. A more complete experiment is now in progress.

Initially a power-spectrum sum of the terms of Eq. (10), which gives the same intensity correlation peak for each satellite in Fig. 3(a), is calculated:

$$
\hat{I}_{\text {sum }}=\Sigma \hat{I}_{b}^{(1)}(u, v) \text {. }
$$

In this way we obtain with one recognition process the simultaneous detection of the three objects presented in Figs. 3 and 4.

A schematic diagram of the optical setup used in these experiments is presented in Fig. 5. The first case consists of recording of power spectrum $\hat{I}_{\text {sum }}$ by means of a photographic register in order to avoid the loss of contrast, resolution, and high-frequency cutoff in the liquid-crystal devices. The slide was recorded in nearly linear conditions.

Figure 6(a) shows the correlation obtained when $\hat{I}_{\text {sum }}$ is placed in front of the lens. As can be observed, the height of the peak corresponding to satellite 3 is slightly lower than foreseen in the previous simulation. The difference is because the recording process is not perfectly linear, and as a consequence the balance between $\hat{I}_{b}^{(1)}(u, v)$ terms is not the same as previously calculated.

In the second case, power spectrum $\hat{I}_{\text {sum }}$ was displayed on a black-and-white liquid-crystal television (LCTV); the correlations obtained are shown in Fig. 6(b). It is clearly observable that the height differences between peaks are severe, and they are wider as a consequence of the loss of high frequencies. ${ }^{13,14}$ Moreover, nonlinearities in the transmission and the low number of gray levels and pixels in the LCTV mean that the power spectrum $\hat{I}_{\text {sum }}$ displayed is significantly different than that previously computed. These results show that it is important to control the whole experimental setup in order to compensate for the nonlinearities and the limitations that alter the balance of Eq. (11).

\section{Conclusions}

A method to control the recognition peaks obtained in a binary joint transform correlator has been presented. This control is obtained by use of the frequencyvariant threshold and a suitable weight function.
This factor is the inverse median value of the amplitude Fourier transform of the reference to be detected. As a consequence, the detection threshold of a device that registers the correlation can be programmed, avoiding missed detection and false alarms. The method has been tested by means of digital simulation, giving fine results.

A simple hybrid setup with a LCTV, which displays the modified power spectrum, and a Fourier lens, has been implemented. In this case the results show that the limitations of the devices are critical.

This study was supported in part by the Spanish Comisión Interministerial de Ciencia y Tecnologia, project ROB91-0554.

\section{References}

1. C. S. Weaver and J. W. Goodman, "A technique for optically convolving two functions," Appl. Opt. 5, 1248-1249 (1966).

2. F. T. S. Yu and H. J. Lu, "A real-time programmable joint transform correlator," Opt. Commun. 52, 10-16 (1984).

3. F. T. S. Yu and T. Nagata, "Binary phase-only joint transform correlator," Microwave Opt. Tech. Lett. 2, 15-19 (1989).

4. B. Javidi, "Nonlinear joint power spectrum based optical correlation," Appl. Opt. 28, 2358-2367 (1989).

5. B. Javidi and J. Wang, "Binary nonlinear joint transform correlation with median and subset median thresholding," Appl. Opt. 30, 967-976 (1991).

6. B. Javidi, J. Wang, and Q. Tang, "Multiple-object binary joint transform correlation using multiple-level threshold crossing," Appl. Opt. 30, 4234-4244 (1991).

7. S. Vallmitjana, I. Juvells, and A. Carnicer, "Evaluation of a suitable threshold for binarization of power spectrum in a noise-free joint transform correlator," Opt. Commun. 90, 221-226 (1992).

8. F. T.S. Yu, F. Cheng, T. Nagata, and D. A. Gregory, "Effects of fringe binarization of multiobject joint transform correlation," Appl. Opt. 28, 2988-2990 (1989).

9. J. A. Davis, E. A. Merrill, D. M. Cotrell, and R. M. Bunch, "Effects of sampling and binarization in the output of the joint transform correlator," Opt. Eng. 29, 1094-1100 (1990).

10. A. Carnicer, I. Juvells, and S. Vallmitjana, "Effects of thresholding level variation in fringe binarization of multiobject joint transform correlation," Appl. Opt. 31, 1012-1014 (1992).

11. F. Cheng, P. Andres, and F. T. S. Yu, "Removal of intraclass associations in joint transform power spectrum," Opt. Commun. 99, 7-12 (1993).

12. F. T. S. Yu, S. Jutamulia, T. W. Lin, and D. A. Gregory, "Adaptive real-time pattern recognition using a liquid crystal TV based joint transform correlator," Appl. Opt. 26, 13701372 (1987).

13. I. Juvells, A. Carnicer, and S. Vallmitjana, "Comparison between experiment and simulation in a real-time joint transform correlator," Jpn. J. Appl. Phys. 31, 1076-1077 (1992).

14. A. Carnicer, I. Juvells, S. Vallmitjana, and J. R. de F. Moneo, "Analysis by digital simulation of the experimental features in real-time joint transform correlator," J. Opt. (Paris) 23, 63-70 (1992). 